\title{
Stochastic quasilinear viscoelastic wave equation with nonlinear damping and source terms
}

\author{
Sangil Kim', Jong-Yeoul Park ${ }^{2}$ and Yong Han Kang ${ }^{3 *}$ [D
}

\section{"Correspondence:}

yonghann@cu.ac.kr

${ }^{3}$ Institute of Liberal Education,

Catholic University of Daegu,

Gyeongsan, South Korea

Full list of author information is

available at the end of the article
The Author(s) 2018. This article is distributed under the terms of the Creative Commons Attribution 4.0 International License (http://creativecommons.org/licenses/by/4.0/), which permits unrestricted use, distribution, and reproduction in any medium, provided you give appropriate credit to the original author(s) and the source, provide a link to the Creative Commons license, and indicate if changes were made.

\begin{abstract}
The goal of this study is to investigate an initial boundary value problem for the stochastic quasilinear viscoelastic wave equation involving the nonlinear damping $\left|u_{t}\right|^{q-2} u_{t}$ and a source term of the type $|u|^{p-2} u$ driven by additive noise. By an appropriate energy inequality, we prove that finite time blow-up is possible for equation (1.1) below if $p>\{q, \rho+2\}$ and the initial data are large enough (that is, if the initial energy is sufficiently negative). Also, we show that if $q \geq p$, the local solution can be extended for all time and is thus global.
\end{abstract}

MSC: $60 \mathrm{H} 15 ; 35 \mathrm{~L} 05 ; 35 \mathrm{~L} 70$

Keywords: stochastic quasilinear viscoelastic wave equation; blow-up of solution; global existence

\section{Introduction}

In this paper, we are concerned with the following stochastic viscoelastic wave equation:

$$
\begin{aligned}
& \left|u_{t}\right|^{\rho} u_{t t}-\Delta u-\Delta u_{t t}+\int_{0}^{t} h(t-\tau) \Delta u(\tau) d \tau+\left|u_{t}\right|^{q-2} u_{t} \\
& \quad=|u|^{p-2} u+\epsilon \sigma(x, t) \partial_{t} W(x, t) \quad \text { in } D \times(0, T), \\
& u=0 \quad \text { on } \partial D \times(0, T), \\
& u(x, 0)=u_{0}(x), \quad u_{t}(x, 0)=u_{1}(x) \quad \text { in } \bar{D},
\end{aligned}
$$

where $D$ is a bounded domain in $\mathbb{R}^{n}$ with smooth boundary $\partial D$, with given positive constants $\rho>0, q \geq 2$, and $p \geq 2$. The function $h: \mathbb{R}^{+} \rightarrow \mathbb{R}^{+}$in the viscoelastic term is a positive relaxation function satisfying some conditions to be specified later. $W(x, t)$ is an infinite dimensional Wiener process, $\sigma(x, t)$ is $L^{2}(D)$-valued progressively measurable, and $\epsilon$ is a given positive constant which measures the strength of noise.

System (1.1) without the stochastic term is a model for quasilinear viscoelastic wave equation with nonlinear damping and source terms. Various forms of the deterministic system (1.1) have been considered by many authors, and several results considering existence, nonexistence, and asymptotic behavior have been established in [1-5], and the references therein. For example, Liu [3] considered the following quasilinear viscoelastic 
wave equation problem:

$$
\begin{aligned}
& \left|u_{t}\right|^{\rho} u_{t t}-\Delta u-\Delta u_{t t}+\int_{0}^{t} g(t-\tau) \Delta u(\tau) d \tau=b|u|^{p-2} u \quad \text { in } D \times(0, \infty), \\
& u=0 \quad \text { on } \partial D \times(0, \infty), \\
& u(x, 0)=u_{0}(x), \quad u_{t}(x, 0)=u_{1}(x) \quad \text { in } \Omega,
\end{aligned}
$$

where $D$ is a bounded domain in $\mathbb{R}^{n}(n \geq 1)$ with a smooth boundary $\partial D$, and $\rho, b>0$, $p>2$ are constants. The author investigated the general solution and blow-up solutions for this problem. Also, Song [4] studied the nonlinear quasilinear viscoelastic wave equation problem

$$
\begin{aligned}
& \left|u_{t}\right|^{\rho} u_{t t}-\Delta u+\int_{0}^{t} g(t-\tau) \Delta u(\tau) d \tau+\left|u_{t}\right|^{m-2} u_{t}=|u|^{p-2} u \quad \text { in } D \times[0, T], \\
& u=0 \quad \text { on } \partial D \times[0, T], \\
& u(x, 0)=u_{0}(x), \quad u_{t}(x, 0)=u_{1}(x) \text { in } D,
\end{aligned}
$$

where $D$ is a bounded domain of $\mathbb{R}^{n}(n \geq 1)$ with a smooth boundary $\partial D, m>2, g: \mathbb{R}^{+} \rightarrow$ $\mathbb{R}^{+}$is a positive nonincreasing function, and

$$
\begin{aligned}
& 2<p<\infty \quad \text { if } n=1,2, \quad 2<p<2(n-1) /(n-2) \quad \text { if } n \geq 3, \\
& 2<\rho<\infty \quad \text { if } n=1,2, \quad 2<\rho \leq n /(n-2) \quad \text { if } n \geq 3 .
\end{aligned}
$$

He proved the global nonexistence of positive initial energy solutions for a quasilinear viscoelastic wave equation.

Under the consideration of random environment, there are many studies on the stochastic wave equation with global existence and invariant measures for linear and nonlinear damping (see the references in [6-25]).

Wei and Jiang [26] and Gao, Guo and Liang [24] considered the following nonlinear stochastic viscoelastic wave equation:

$$
\begin{aligned}
& u_{t t}-\Delta u+\int_{0}^{t} h(t-\tau) \Delta u(\tau) d \tau+u_{t} \\
& \quad=|u|^{p-2} u+\epsilon \sigma(u, \nabla u, x, t) \partial_{t} W(x, t) \quad \text { in } D \times(0, T), \\
& u=0 \quad \text { on } \partial D \times(0, T), \\
& u(x, 0)=u_{0}(x), \quad u_{t}(x, 0)=u_{1}(x) \quad \text { in } \bar{D} .
\end{aligned}
$$

They investigated the global existence and the energy decay estimate of a solution and showed that the solution blows up with positive probability or it is explosive in $L^{2}$ sense under some conditions.

Moreover, Cheng et al. [23] proved the existence of a global solution and blow-up solutions with positive probability for the nonlinear stochastic viscoelastic wave equation with linear damping (see $[18,22,26])$. 
Recently, Cheng et al. [23] studied the stochastic viscoelastic wave equation with nonlinear damping and source terms

$$
\begin{aligned}
& u_{t t}-\Delta u+\int_{0}^{t} h(t-\tau) \Delta u(\tau) d \tau+\left|u_{t}\right|^{q-2} u_{t} \\
& \quad=|u|^{p-2} u+\epsilon \sigma(x, t) \partial_{t} W(x, t) \quad \text { in } D \times(0, T), \\
& u=0 \quad \text { on } \partial D \times(0, T), \\
& u(x, 0)=u_{0}(x), \quad u_{t}(x, 0)=u_{1}(x) \quad \text { in } \bar{D},
\end{aligned}
$$

where $D$ is a bounded domain in $\mathbb{R}^{n}$ with smooth boundary $\partial D, q \geq 2, p \geq 2, \epsilon$ is a given positive constant which measures the strength of noise; $W(x, t)$ is an infinite dimensional Wiener process; $\sigma(x, t, w)$ is $L^{2}(D)$-valued progressively measurable; and $h$ is a positive relaxation function. The authors studied the global solution of stochastic viscoelastic wave equations with nonlinear damping and source terms.

The previous work in Cheng et al. [23] established that the solution blows up with positive probability or it is explosive in energy sense for $p>q$. Motivated by this work, we prove that the stochastic quasilinear viscoelastic wave equation (1.1) can blow up with positive probability or it is explosive in energy sense for $p>\{q, \rho+2\}$ and obtain the existence of global solution by the Borel-Cantelli lemma. To the best of our knowledge, there have been no results for the blow-up of solutions of stochastic quasilinear viscoelastic wave equation with positive probability.

This paper is organized as follows. In Section 2, we present some assumptions, definitions, and lemmas needed for our work. The result for the local existence and a pointwise unique solution of equation (1.1) are given too. In Section 3, we show Lemmas 3.1 and 3.2. With those lemmas, we prove our main result for $p>\{q, \rho+2\}$. In Section 4 , we obtain global existence of equation (1.1).

\section{Preliminaries}

Let $\left(X,\|\cdot\|_{X}\right)$ be a separable Hilbert space with Borel $\sigma$-algebra $\mathbf{B}(X)$, and let $(\Omega, \mathfrak{F}, P)$ be a probability space. We set $H=L^{2}(D)$ with the inner product and norm denoted by $(\cdot, \cdot)$ and $\|\cdot\|$, respectively. We denote by $\|\cdot\|_{q}$ the $L^{q}(D)$ norm for $0 \leq q \leq \infty$ and by $\|\nabla \cdot\|$ the Dirichlet norm in $V=H_{0}^{1}(D)$ which is equivalent to $H^{1}(D)$ norm.

First, we introduce the following hypotheses:

(H1) We assume that $p, q, \rho$ satisfy

$$
\begin{aligned}
& q \geq 2, \quad p>2, \quad \max \{p, q\} \leq \frac{2(n-1)}{n-2} \quad \text { if } n \geq 3 \\
& q \geq 2, \quad p>2 \quad \text { if } n=1,2 \\
& 0 \leq \rho \leq \frac{2}{n-2} \quad \text { if } n \geq 3, \quad 0<\rho<\infty \quad \text { if } n=1,2 .
\end{aligned}
$$

(H2) We assume that $h: \mathbb{R}^{+} \rightarrow \mathbb{R}^{+}$is a bounded nonincreasing $C^{1}$ function satisfying

$$
h(s)>0, \quad 1-\int_{0}^{\infty} h(s) d s=l>0,
$$


and there exist positive constants $\xi_{1}$ and $\xi_{2}$ such that

$$
-\xi_{1} h(t) \leq h^{\prime}(t) \leq-\xi_{2} h(t), \quad t \geq 0 .
$$

(H3) $\sigma(x, t)$ is $H_{0}^{1}(D) \cap L^{\infty}(D)$-valued progressively measurable such that

$$
E \int_{0}^{T}\left(\|\nabla \sigma(t)\|^{2}+\|\sigma(t)\|_{\infty}^{2}\right) d t<\infty
$$

Lemma 2.1 ([8]) For all $u, v \in H^{1}\left(\mathbb{R}^{n}\right)$ and $0<p \leq \frac{2}{n-2}(n \geq 3)$ or $p>0(n=1,2)$, there exists a constant $c_{1}=c_{1}(n, p)>0$ such that

$$
\|u\|_{L^{2(p-1)}} \leq c_{1}\|u\|_{H^{1}}, \quad\left\|u^{p} v\right\| \leq c_{1}^{p+1}\|u\|_{H^{1}}^{p}\|v\|_{H^{1}} .
$$

In this paper, $E(\cdot)$ stands for expectation with respect to probability measure $P$, and $W(x, t)(t \geq 0)$ is a $V$-valued $Q$-Wiener process on the probability space with the covariance operator $Q$ satisfying $\operatorname{Tr}(Q)<\infty$. A complete orthonormal system $\left\{e_{k}\right\}_{k=1}^{\infty}$ in $V$ with $c_{0}:=$ $\sup _{k \geq 1}\left\|e_{k}\right\|_{\infty}<\infty$ and a bounded sequence of nonnegative real members $\left\{\lambda_{k}\right\}_{k=1}^{\infty}$ satisfy that

$$
Q e_{k}=\lambda_{k} e_{k}, \quad k=1,2, \ldots
$$

To simplify the computations, we assume that the covariance operator $Q$ and Laplacian $-\triangle$ with a homogeneous Dirichlet boundary condition have a common set of eigenfunctions, that is,

$$
\begin{aligned}
& -\triangle e_{k}=\mu_{k} e_{k}, \quad x \in D, \\
& e_{k}=0, \quad x \in \partial D,
\end{aligned}
$$

and then, for any $t \in[0, T], W(x, t)$ has an expansion

$$
W(x, t)=\sum_{k=1}^{\infty} \sqrt{\lambda_{k}} \beta_{k}(t) e_{k}(t)
$$

where $\left\{\beta_{k}(t)\right\}_{k=1}^{\infty}$ are real-valued Brownian motions mutually independent of $(\Omega, \mathfrak{F}, P)$. Let $\mathcal{H}$ be the set of $L_{2}^{0}=L^{2}\left(Q^{1 / 2} V, V\right)$-valued processes with the norm

$$
\|\Phi(t)\|_{\mathcal{H}}=\left(E \int_{0}^{t}\|\Phi(s)\|_{L_{2}^{0}}^{2} d s\right)^{1 / 2}=\left(E \int_{0}^{t} \operatorname{Tr}\left(\Phi(s) Q \Phi^{*}(s)\right) d s\right)^{1 / 2}<\infty
$$

where $\Phi^{*}(s)$ denotes the adjoint operator of $\Phi(s)$. For any $\Phi^{*}(t) \in \mathcal{H}$, we can define the stochastic integral with respect to the $Q$-Wiener process as $\int_{0}^{t} \Phi(s) d W(s)$, which is martingale. For more details about the finite dimension Winner process and the stochastic integral, see [22].

Definition 2.1 Assume that $\left(u_{0}, u_{1}\right) \in H_{0}^{1}(D) \times L^{2}(D)$ and $E \int_{0}^{T}\|\sigma(t)\|^{2} d t<\infty$. $u$ is said to be the solution of (1.1) on the interval $[0, T]$ if $\left(u, u_{t}\right)$ is $H_{0}^{1}(D) \times L^{2}(D)$-valued progressively 
measurable, $\left(u, u_{t}\right) \in L^{2}\left(\Omega ; C\left([0, T] ; H_{0}^{1}(D) \times L^{2}(D)\right)\right), u_{t} \in L^{q}((0, T) \times D)$, and such that (1.1) holds in the sense of distributions over $(0, T) \times D$ for almost all $w$.

By combining the arguments of $[20,23,24]$, we get the existence result.

Theorem 2.1 ([20, 24]) Assume that (H1)-(H3) hold. Then, for the initial data $\left(u_{0}, u_{1}\right) \in$ $\left(H^{2}(D) \cap H_{0}^{1}(D)\right) \times H_{0}^{1}(D)$, problem (1.1) has a pointwise unique solution $u$ such that

$$
u \in L^{2}\left(\Omega ; L^{\infty}\left(0, T ; H^{2}(D) \cap H_{0}^{1}(D)\right)\right) \cap L^{2}\left(\Omega ; C\left([0, T] ; H_{0}^{1}(D)\right)\right)
$$

and

$$
u_{t} \in L^{2}\left(\Omega ; L^{\infty}\left(0, T ; H_{0}^{1}(D)\right)\right) \cap L^{2}\left(\Omega ; C\left([0, T] ; L^{2}(D)\right)\right) .
$$

\section{Blow-up result}

In this section, we prove our main result for $p>q$. For this purpose, we give defined restrictions on $\sigma(x, t)$ and the relaxation function $h$ such that

$$
E \int_{0}^{\infty} \int_{D} \sigma^{2}(x, t) d x d t<\infty, \quad \int_{0}^{\infty} h(s) d s<\frac{p(p-2)}{(p-1)^{2}}
$$

Now, we define an energy function

$$
\begin{aligned}
F(t)= & \frac{1}{\rho+2}\left\|u_{t}(t)\right\|_{\rho+2}^{\rho+2}+\frac{1}{2}\left\|\nabla u_{t}(t)\right\|^{2} \\
& +\frac{1}{2}\left(1-\int_{0}^{t} h(s) d s\right)\|\nabla u(t)\|^{2}+\frac{1}{2}(h \circ \nabla u)(t)-\frac{1}{p}\|u(t)\|_{p}^{p},
\end{aligned}
$$

where

$$
(h \circ \nabla u)(t)=\int_{0}^{t} h(t-s)\|\nabla u(t)-\nabla u(s)\|^{2} d s
$$

For each $N$, stopping time $\tau_{N}$ is given as

$$
\tau_{N}=\inf \left\{t>0:\|\nabla u(t)\|^{2} \geq N\right\}
$$

where $\tau_{N}$ is increasing in $N$, and $\tau_{\infty}=\lim _{N \rightarrow \infty} \tau_{N}$. In order to prove our blow-up result, we rewrite (1.1) as an equivalent Itô's system

$$
\begin{aligned}
& d u=v d t, \\
& d\left(\frac{1}{\rho+1}|v|^{\rho} v-\Delta v\right)=\left(\Delta u-\int_{0}^{t} h(t-s) \Delta u(s) d s-|v|^{q-2} v+|u|^{p-2} u\right) d t \\
& +\epsilon \sigma(x, t) d W_{t}(x, t), \quad(x, t) \in D \times(0, T), \\
& u(x, t)=0, \quad(x, t) \in \partial D \times(0, T), \\
& u(x, 0)=u_{0}(x), \quad v(x, 0)=v_{0}(x)=u_{1}(x), \quad x \in D,
\end{aligned}
$$


where $\left(u_{0}, u_{1}\right) \in H_{0}^{1}(D) \times L^{2}(D)$. Then the energy function $F(t)$ becomes

$$
\begin{aligned}
F(t)= & \frac{1}{\rho+2}\|v(t)\|_{\rho+2}^{\rho+2}+\frac{1}{2}\|\nabla v(t)\|^{2} \\
& +\frac{1}{2}\left(1-\int_{0}^{t} h(s) d s\right)\|\nabla u(t)\|^{2}+\frac{1}{2}(h \circ \nabla u)(t)-\frac{1}{p}\|u(t)\|_{p}^{p} .
\end{aligned}
$$

Lemma 3.1 Let $(u, v)$ be a solution of Eq. (3.3) with the initial data $\left(u_{0}, v_{0}\right) \in H_{0}^{1}(D) \times$ $L^{2}(D)$. Then we have

$$
\begin{aligned}
\frac{d}{d t} E F(t)= & -E\|v(t)\|_{q}^{q}+\frac{\epsilon^{2}}{2} \sum_{j=1}^{\infty} E \int_{D} \lambda_{j} e_{j}^{2}(x) \sigma^{2}(x, t) d x \\
& -E\left(-h^{\prime} \circ \nabla u\right)(t)-\frac{1}{2} h(t) E\|\nabla u(t)\|^{2} \\
\leq & -E\|v(t)\|_{q}^{q}+\frac{\epsilon^{2}}{2} \sum_{j=1}^{\infty} E \int_{D} \lambda_{j} e_{j}^{2}(x) \sigma^{2}(x, t) d x,
\end{aligned}
$$

and

$$
\begin{aligned}
& E\left(u(t), \frac{1}{\rho+1}|v(t)|^{\rho} v(t)-\Delta v(t)\right) \\
& =\left(u_{0}, \frac{1}{\rho+1}\left|u_{1}\right|^{\rho} u_{1}-\Delta u_{1}\right)-\int_{0}^{t} E\|\nabla u(s)\|^{2} d s \\
& \quad-\int_{0}^{t} E\left(u(s),|v(s)|^{q-2} v(s)\right) d s+\int_{0}^{t} E\|u(s)\|_{p}^{p} d s \\
& \quad+E \int_{0}^{t} \int_{0}^{s} h(s-\tau)(\nabla u(\tau), \nabla u(s)) d \tau d s \\
& \quad+\frac{1}{\rho+1} E \int_{0}^{t}\|v(s)\|_{\rho+2}^{\rho+2} d s+E \int_{0}^{t}\|\nabla v(s)\|^{2} d s .
\end{aligned}
$$

Proof By multiplying Eq. (3.3) by $v(t)$ and using Itô's formula, we deduce (3.5). Also, multiplying Eq. (3.3) by $u(t)$ and integrating by parts over $(0, T)$, we arrive at (3.6) (see [24]).

Let

$$
G(t)=\frac{\epsilon^{2}}{2} \sum_{j=1}^{\infty} E \int_{0}^{t} \int_{D} \lambda_{j} e_{j}^{2}(x) \sigma^{2}(x, s) d x d s .
$$

Due to (3.1), we deduce

$$
\begin{aligned}
G(\infty) & =\frac{\epsilon^{2}}{2} \sum_{j=1}^{\infty} E \int_{0}^{\infty} \int_{D} \lambda_{j} e_{j}^{2}(x) \sigma^{2}(x, s) d x d s \\
& \leq \frac{\epsilon^{2}}{2} \operatorname{Tr}(Q) c_{0}^{2} E \int_{0}^{\infty} \int_{D} \sigma^{2}(x, s) d x d s=E_{1}<\infty .
\end{aligned}
$$


We set

$$
H(t)=G(t)-E[F(t)]
$$

Then (3.5) implies that

$$
H^{\prime}(t)=G^{\prime}(t)-\frac{d}{d t} E[F(t)] \geq E\|v(t)\|_{q}^{q} \geq 0 .
$$

Lemma 3.2 Let $(u, v)$ be a solution of Eq. (3.3). Then there exists a positive constant $C$ such that

$$
\begin{aligned}
E\|u(t)\|_{p}^{s} \leq & C\left[G(t)-H(t)-E\|v(t)\|_{\rho+2}^{\rho+2}\right. \\
& \left.-E\|\nabla v(t)\|^{2}+E\|u(t)\|_{p}^{p}-E(h \circ \nabla u)(t)\right], \quad 2 \leq s \leq p .
\end{aligned}
$$

Proof If $\|u\|_{p} \leq 1$, then $\|u\|_{p}^{s} \leq\|u\|_{p}^{2} \leq C\|\nabla u\|^{2}$ by the Sobolev embedding theorem. If $\|u\|_{p} \geq 1$, then $\|u\|_{p}^{s} \leq\|u\|_{p}^{p}$. Thus there exists a constant $C>0$ such that $E\|u\|_{p}^{s} \leq$ $C\left(E\|\nabla u\|^{2}+E\|u\|_{p}^{p}\right)$. Therefore, combining with the definition of energy function, we get (3.10).

Theorem 3.1 Assume that (H1)-(H3) and (3.1) hold. Let $(u, v)$ be a solution of Eq. (3.3) with the initial data $\left(u_{0}, v_{0}\right) \in H_{0}^{1}(D) \times L^{2}(D)$ satisfying

$$
F(0) \leq-(1+\beta) E_{1},
$$

where $\beta>0$ is an arbitrary constant and $E_{1}$ is defined in (3.8). If $p>\{q, \rho+2\}$, then the solution $(u, v)$ and the lifespan $\tau_{\infty}$ defined above are either

(1) $P\left(\tau_{\infty}<\infty\right)>0$, that is, $\|\nabla u(t)\|$ blows up in finite time with positive probability, or

(2) there exists a positive time $T^{*} \in\left[0, T_{0}\right]$ such that

$$
\lim _{t \rightarrow T^{*}} E[F(t)]=+\infty
$$

where

$$
\begin{aligned}
& T_{0}=\frac{1-\alpha}{\alpha K L^{\alpha /(1-\alpha)}(0)}, \\
& L(0)=H^{1-\alpha}(0)+\delta E\left(u_{0}, \frac{1}{\rho+1}\left|u_{1}\right|^{\rho} u_{1}-\Delta u_{1}\right)>0,
\end{aligned}
$$

and $\alpha, K$ are given later.

Proof For the lifespan $\tau_{\infty}$ of the solution $\{u(t): t>0\}$ of Eq. (3.3) with $H_{0}^{1}(D)$ norm, we treat the case when $P\left(\tau_{\infty}=+\infty\right)<1$. Then, for sufficiently large $T>0$, by (3.9) and (3.11), we obtain

$$
0<(1+\beta) E_{1} \leq-F(0)=H(0) \leq H(t) \leq G(t)+\frac{1}{p} E\|u(t)\|_{p}^{p} \leq E_{1}+\frac{1}{p} E\|u(t)\|_{p}^{p} .
$$


Define

$$
L(t)=H^{1-\alpha}(t)+\delta E\left(u(t), \frac{1}{\rho+1}|v(t)|^{\rho} v(t)-\Delta v(t)\right),
$$

where

$$
0<\alpha<\min \left\{\frac{1}{2}, \frac{p-2}{2 p}, \frac{p-q}{p q}, \frac{1}{\rho+2}-\frac{1}{p}\right\}
$$

and $\delta$ is a very small constant to be determined later.

Using (3.6) and (3.9), we deduce

$$
\begin{aligned}
L^{\prime}(t)= & (1-\alpha) H^{-\alpha}(t) H^{\prime}(t)+\delta\left[-E\|\nabla u(t)\|^{2}-E\left(u(t),|v(t)|^{q-2} v(t)\right)\right. \\
& +E\|u(t)\|_{p}^{p}+E \int_{0}^{t} h(t-\tau)(\nabla u(\tau), \nabla u(t)) d \tau \\
& \left.+\frac{1}{\rho+1} E\|v(t)\|_{\rho+2}^{\rho+2}+E\|\nabla v(t)\|^{2}\right] \\
\geq & (1-\alpha) H^{-\alpha}(t) E\|v(t)\|_{q}^{q}+\delta p[H(t)-G(t)+E F(t)] \\
& -\delta E\|\nabla u(t)\|^{2}-\delta E\left(u(t),|v(t)|^{q-2} v(t)\right)+\delta E\|u(t)\|_{p}^{p} \\
& +\delta E \int_{0}^{t} h(t-\tau)(\nabla u(\tau), \nabla u(t)) d \tau+\frac{\delta}{\rho+1} E\|v(t)\|_{\rho+2}^{\rho+2}+\delta E\|\nabla v(t)\|^{2} \\
\geq & (1-\alpha) H^{-\alpha}(t) E\|v(t)\|_{q}^{q}+\delta p H(t) \\
& +\delta\left(\frac{p}{\rho+2}+\frac{1}{\rho+1}\right) E\|v(t)\|_{\rho+2}^{\rho+2} \\
& +\delta\left(\frac{p}{2}-1\right) E\|\nabla u(t)\|^{2} \\
& +\delta\left(\frac{p}{2}+1\right) E\|\nabla v(t)\|^{2}-\delta E\left(u(t),|v(t)|^{q-2} v(t)\right) \\
& +\delta E \int_{0}^{t} h(t-\tau)(\nabla u(\tau), \nabla u(t)) d \tau \\
& +\frac{\delta p}{2} E(h \circ \nabla u)(t)-\frac{\delta p}{2} E \int_{0}^{t} h(\tau) d \tau\|\nabla u(t)\|^{2}-\delta p G(t) . \\
&
\end{aligned}
$$

On the other hand, we have

$$
\begin{aligned}
& \delta E \int_{0}^{t} h(t-\tau)(\nabla u(\tau), \nabla u(t)) d \tau \\
& =\delta E \int_{0}^{t} h(t-\tau)(\nabla u(\tau)-\nabla u(t), \nabla u(t)) d \tau \\
& \quad+\delta E \int_{0}^{t} h(\tau) d \tau\|\nabla u(t)\|^{2},
\end{aligned}
$$


and by Hölder's inequality, we get

$$
\begin{aligned}
& \delta E \int_{0}^{t} h(t-\tau)(\nabla u(\tau)-\nabla u(t), \nabla u(t)) d \tau \\
& \quad \geq-\frac{\delta p}{2} E(h \circ \nabla u)(t)-\frac{\delta}{2 p} E \int_{0}^{t} h(\tau) d \tau\|\nabla u(t)\|^{2} .
\end{aligned}
$$

Inserting (3.17) and (3.18) into (3.16), we obtain

$$
\begin{aligned}
L^{\prime}(t) \geq & (1-\alpha) H^{-\alpha}(t) E\|v(t)\|_{q}^{q}+\delta p H(t) \\
& +\delta\left(\frac{p}{\rho+2}+\frac{1}{\rho+1}\right) E\|v(t)\|_{\rho+2}^{\rho+2} \\
& +\delta\left(\frac{p}{2}-1\right) E\|\nabla u(t)\|^{2} \\
& +\delta\left(\frac{p}{2}+1\right) E\|\nabla v(t)\|^{2}-\delta E\left(u(t),|v(t)|^{q-2} v(t)\right) \\
& -\delta p G(t)+\delta\left(1-\frac{p^{2}+1}{2 p}\right) E \int_{0}^{t} h(\tau) d \tau\|\nabla u(t)\|^{2} .
\end{aligned}
$$

For $q<p$, by $E\|u(t)\|_{q}^{q} \leq c E\|u(t)\|_{p}^{q}$ and Hölder's inequality, we deduce the following estimate (see [23]):

$$
\begin{aligned}
E\left(u(t),|v(t)|^{q-2} v(t)\right) & \leq\left(E\|v(t)\|_{q}^{q}\right)^{\frac{q-1}{q}}\left(E\|u(t)\|_{q}^{q}\right)^{\frac{1}{q}} \\
& \leq C\left(E\|v(t)\|_{q}^{q}\right)^{\frac{q-1}{q}}\left(E\|u(t)\|_{p}^{q}\right)^{\frac{1}{q}} \\
& \leq C\left(E\|v(t)\|_{q}^{q}\right)^{\frac{q-1}{q}}\left(E\|u(t)\|_{p}^{p}\right)^{\frac{1}{p}} \\
& \leq C\left(E\|v(t)\|_{q}^{q}\right)^{\frac{q-1}{q}}\left(E\|u(t)\|_{p}^{p}\right)^{\frac{1}{q}}\left(E\|u(t)\|_{p}^{p}\right)^{\frac{1}{p}-\frac{1}{q}}
\end{aligned}
$$

and Young's inequality

$$
\left(E\|v(t)\|_{q}^{q}\right)^{\frac{q-1}{q}}\left(E\|u(t)\|_{p}^{p}\right)^{\frac{1}{q}} \leq \frac{q-1}{q} \mu E\|v(t)\|_{q}^{q}+\frac{\mu^{1-q}}{q} E\|u(t)\|_{p}^{p},
$$

where $\mu$ is a constant to be determined later. In view of (3.14), we get

$$
E\|u(t)\|_{p}^{p} \geq p(H(t)-G(t)) \geq \widetilde{\rho} H(t)
$$

where $\widetilde{\rho}=p \beta /(1+\beta)$. With the assumption of $H(0)>1,(3.21),(3.22)$, and (3.15) imply that

$$
\left(E\|u(t)\|_{p}^{p}\right)^{\frac{1}{p}-\frac{1}{q}} \leq \widetilde{\rho}^{\frac{1}{p}-\frac{1}{q}} H(t)^{\frac{1}{p}-\frac{1}{q}} \leq \widetilde{\rho}^{\frac{1}{p}-\frac{1}{q}} H^{-\alpha}(t) \leq \widetilde{\rho}^{\frac{1}{p}-\frac{1}{q}} H^{-\alpha}(0) .
$$

Combining with (3.20), (3.21), and (3.23), we arrive at

$$
\left|E\left(u(t),|v(t)|^{q-2} v(t)\right)\right| \leq a_{1} \frac{q-1}{q} \mu E\|v(t)\|_{q}^{q} H^{-\alpha}(t)+a_{1} \frac{\mu^{1-q}}{q} E\|u(t)\|_{p}^{p} H^{-\alpha}(t),
$$


where $a_{1}=C \widetilde{\rho}^{\frac{1}{p}-\frac{1}{q}}$. Hence, substituting (3.24) for (3.19), we get

$$
\begin{aligned}
L^{\prime}(t) \geq & \left(1-\alpha-a_{1} \frac{q-1}{q} \mu \delta\right) H^{-\alpha}(t) E\|v(t)\|_{q}^{q}+\delta p H(t) \\
& +\delta\left(\frac{p}{\rho+2}+\frac{1}{\rho+1}\right) E\|v(t)\|_{\rho+2}^{\rho+2} \\
& +\delta\left(\frac{p}{2}-1\right) E\|\nabla u(t)\|^{2} \\
& +\delta\left(\frac{p}{2}+1\right) E\|\nabla v(t)\|^{2}-\delta p G(t) \\
& +\delta\left(1-\frac{p^{2}+1}{2 p}\right) \int_{0}^{t} h(\tau) d \tau E\|\nabla u(t)\|^{2} \\
& -\delta a_{1} \frac{\mu^{1-q}}{q} E\|u(t)\|_{p}^{p} H^{-\alpha}(0) .
\end{aligned}
$$

Using Lemma 3.2 with $s=p$ and (3.25), we have

$$
\begin{aligned}
L^{\prime}(t) \geq & \left(1-\alpha-a_{1} \frac{q-1}{q} \mu \delta\right) H^{-\alpha}(t) E\|v(t)\|_{q}^{q}+\delta p H(t) \\
& +\delta\left(\frac{p}{\rho+2}+\frac{1}{\rho+1}\right) E\|v(t)\|_{\rho+2}^{\rho+2} \\
& +\delta\left(\frac{p}{2}-1\right) E\|\nabla u(t)\|^{2} \\
& +\delta\left(\frac{p}{2}+1\right) E\|\nabla v(t)\|^{2}-\delta p G(t) \\
& +\delta\left(1-\frac{p^{2}+1}{2 p}\right) E \int_{0}^{t} h(\tau) d \tau\|\nabla u(t)\|^{2} \\
& -\delta a_{2} \mu^{1-q}\left[G(t)-H(t)-E\|v(t)\|_{\rho+2}^{\rho+2}\right. \\
& \left.-E\|\nabla v(t)\|^{2}+E\|u(t)\|_{p}^{p}-E(h \circ \nabla u)(t)\right] \\
\geq & \left(1-\alpha-a_{1} \frac{q-1}{q} \mu \delta\right) H^{-\alpha}(t) E\|v(t)\|_{q}^{q} \\
& +\delta\left(p+a_{2} \mu^{1-q}\right) H(t)-\delta\left(p+a_{2} \mu^{1-q}\right) G(t) \\
& +\delta\left(\frac{p}{\rho+2}+\frac{1}{\rho+1}+a_{2} \mu^{1-q}\right) E\|v(t)\|_{\rho+2}^{\rho+2} \\
& +\delta\left(\frac{p}{2}+1+a_{2} \mu^{1-q}\right) E\|\nabla v(t)\|^{2}-\delta a_{2} \mu^{1-q} E\|u(t)\|_{p}^{p} \\
& +\delta a_{2} \mu^{1-q} E(h \circ \nabla u)(t) \\
& +\delta\left[\frac{p}{2}-1+\left(1-\frac{p^{2}+1}{2 p}\right) \int_{0}^{t} h(\tau) d \tau\right] E\|\nabla u(t)\|^{2} \\
&
\end{aligned}
$$

where $a_{2}=C a_{1} H^{-\alpha}(0) / q$. 
Note that

$$
\begin{aligned}
H(t) \geq & G(t)+\frac{1}{p} E\|u(t)\|_{p}^{p}-\frac{1}{\rho+2} E\|v(t)\|_{\rho+2}^{\rho+2} \\
& -\frac{1}{2} E\|\nabla v(t)\|^{2}-\frac{1}{2}\|\nabla u(t)\|^{2}-\frac{1}{2} E(h \circ \nabla u)(t)
\end{aligned}
$$

with

$$
a_{3}=\frac{p}{2}-1+\left(1-\frac{p^{2}+1}{2 p}\right) \int_{0}^{t} h(\tau) d \tau>0
$$

we write $p=2 a_{4}+\left(p-2 a_{4}\right)$ with $a_{4}=\min \left\{a_{1}, a_{3}\right\}$, then estimate (3.26) yields

$$
\begin{aligned}
L^{\prime}(t) \geq & \left(1-\alpha-a_{1} \frac{q-1}{q} \mu \delta\right) H^{-\alpha}(t) E\|v(t)\|_{q}^{q} \\
& +\delta\left(p-2 a_{4}+a_{2} \mu^{1-q}\right) H(t)-\delta\left(p-2 a_{4}+a_{2} \mu^{1-q}\right) G(t) \\
& +\delta\left(\frac{p}{\rho+2}-\frac{2 a_{4}}{\rho+2}+\frac{1}{\rho+1}+a_{2} \mu^{1-q}\right) E\|v(t)\|_{\rho+2}^{\rho+2} \\
& +\delta\left(\frac{p}{2}-a_{4}+1+a_{2} \mu^{1-q}\right) E\|\nabla v(t)\|^{2} \\
& +\delta\left(-a_{2} \mu^{1-q}+\frac{2 a_{4}}{p}\right) E\|u(t)\|_{p}^{p} \\
& +\delta\left(a_{2} \mu^{1-q}-a_{4}\right) E(h \circ \nabla u)(t)+\delta\left(a_{3}-a_{4}\right) E\|\nabla u(t)\|^{2} .
\end{aligned}
$$

From (3.8) and (3.14), we deduce

$$
\begin{aligned}
\left(p-2 a_{4}+a_{2} \mu^{1-q}\right) G(t) & \leq\left(p-2 a_{4}+a_{2} \mu^{1-q}\right) E_{1} \\
& \leq \frac{p-2 a_{4}+a_{2} \mu^{1-q}}{1+\beta} H(t) .
\end{aligned}
$$

Substituting (3.30) with (3.29), we get

$$
\begin{aligned}
L^{\prime}(t) \geq & \left(1-\alpha-a_{1} \frac{q-1}{q} \mu \delta\right) H^{-\alpha}(t) E\|v(t)\|_{q}^{q} \\
& +\delta\left(p-2 a_{4}+a_{2} \mu^{1-q}\right) \frac{\beta}{1+\beta} H(t) \\
& +\delta\left(\frac{p}{\rho+2}-\frac{2 a_{4}}{\rho+2}+\frac{1}{\rho+1}+a_{2} \mu^{1-q}\right) E\|v(t)\|_{\rho+2}^{\rho+2} \\
& +\delta\left(\frac{p}{2}-a_{4}+1+a_{2} \mu^{1-q}\right) E\|\nabla v(t)\|^{2} \\
& +\delta\left(-a_{2} \mu^{1-q}+\frac{2 a_{4}}{p}\right) E\|u(t)\|_{p}^{p} \\
& +\delta\left(a_{2} \mu^{1-q}-a_{4}\right) E(h \circ \nabla u)(t)+\delta\left(a_{3}-a_{4}\right) E\|\nabla u(t)\|^{2} .
\end{aligned}
$$


Next, we can choose $\mu$ large enough so that (3.31) becomes

$$
\begin{aligned}
L^{\prime}(t) \geq & \left(1-\alpha-a_{1} \frac{q-1}{q} \mu \delta\right) H^{-\alpha}(t) E\|v(t)\|_{q}^{q}+\delta \gamma\left(H(t)+E\|v(t)\|_{\rho+2}^{\rho+2}+E\|\nabla v(t)\|^{2}\right. \\
& \left.+E\|u(t)\|_{p}^{p}+E(h \circ \nabla u)(t)+E\|\nabla u(t)\|^{2}\right),
\end{aligned}
$$

where

$$
\begin{aligned}
\gamma= & \min \left\{\left(p-2 a_{4}+a_{2} \mu^{1-q}\right) \frac{\beta}{1+\beta}, \frac{p}{\rho+2}-\frac{2 a_{4}}{\rho+2}+\frac{1}{\rho+1}+a_{2} \mu^{1-q},\right. \\
& \left.\frac{p}{2}-a_{4}+1+a_{2} \mu^{1-q},-a_{2} \mu^{1-q}+\frac{2 a_{4}}{p}, a_{2} \mu^{1-q}-a_{4}, a_{3}-a_{4}\right\}>0 .
\end{aligned}
$$

Once $\mu$ is fixed, we pick $\delta$ small enough so that

$$
1-\alpha-a_{1} \frac{q-1}{q} \mu \delta>0
$$

Using this, (3.32) takes the form

$$
\begin{aligned}
L^{\prime}(t) \geq & \delta \gamma\left(H(t)+E\|v(t)\|_{\rho+2}^{\rho+2}+E\|\nabla v(t)\|^{2}\right. \\
& \left.+E\|u(t)\|_{p}^{p}+E(h \circ \nabla u)(t)+E\|\nabla u(t)\|^{2}\right) .
\end{aligned}
$$

Thus, we see that

$$
L(t) \geq L(0)=H^{1-\alpha}(0)+\delta E\left(u_{0}, \frac{1}{\rho+1}\left|u_{1}\right|^{\rho} u_{1}-\Delta u_{1}\right)>0, \quad \forall t \geq 0 .
$$

Consequently, we get

$$
L(t) \geq L(0)>0, \quad \forall t \geq 0 .
$$

Since

$$
\begin{aligned}
\left.\left|E \int_{D}\right| v(t)\right|^{\rho} v(t) u(t) d x \mid & \leq E\|v(t)\|_{\rho+2}^{\rho+1} E\|u(t)\|_{\rho+2} \\
& \leq C E\|v(t)\|_{\rho+2}^{\rho+1} E\|u(t)\|_{p},
\end{aligned}
$$

we have

$$
\begin{aligned}
\left.\left.\left|E \int_{D}\right| v(t)\right|^{\rho} v(t) u(t) d x\right|^{\frac{1}{1-\alpha}} & \leq E\|v(t)\|_{\rho+2}^{\frac{\rho+1}{1-\alpha}} E\|u(t)\|_{\rho+2}^{\frac{1}{1-\alpha}} \\
& \leq C\left[\left(E\|v(t)\|_{\rho+2}^{\rho+2}\right)^{\frac{(\rho+1)}{(\rho+2)(1-\alpha)}} \zeta+\left(E\|u(t)\|_{p}^{2}\right)^{\frac{\theta}{2(1-\alpha)}}\right]
\end{aligned}
$$

where $\frac{1}{\zeta}+\frac{1}{\theta}=1$. By choosing $\zeta=\frac{(1-\alpha)(\rho+2)}{\rho+1}(>1)$, we have $\frac{\theta}{2(1-\alpha)}=\frac{\rho+2}{2[(1-\alpha)(\rho+2)-(\rho+1)]}<\frac{p}{2}$. And with (3.15), (3.36) becomes

$$
\left.\left.\left|E \int_{D}\right| v(t)\right|^{\rho} v(t) u(t) d x\right|^{\frac{1}{1-\alpha}} \leq C\left\{E\|v(t)\|_{\rho+2}^{\rho+2}+\left(E\|u(t)\|_{p}^{2}\right)^{\frac{\rho+2}{2[(1-\alpha)(\rho+2)-(\rho+1)]}}\right\} .
$$


Using Lemma 3.2 with $s=\frac{\rho+2}{2[(1-\alpha)(\rho+2)-(\rho+1)]}$, we obtain

$$
\begin{aligned}
\left.\left.\left|E \int_{D}\right| v(t)\right|^{\rho} v(t) u(t) d x\right|^{\frac{1}{1-\alpha}} \leq & C\left[H(t)+E\|v(t)\|_{\rho+2}^{\rho+2}+E\|\nabla v(t)\|^{2}\right. \\
& \left.+E\|u(t)\|_{p}^{p}+E(h \circ \nabla u)(t)+E\|\nabla u(t)\|^{2}\right] .
\end{aligned}
$$

Therefore, we deduce, for all $t \geq 0$,

$$
\begin{aligned}
L^{\frac{1}{1-\alpha}}(t)= & \left(H^{1-\alpha}(t)+\frac{\delta}{\rho+1} E \int_{D}|v(t)|^{\rho} v(t) u(t) d x+\delta E \int_{D} \nabla v(t) \cdot \nabla u(t) d x\right)^{\frac{1}{1-\alpha}} \\
\leq & C\left[H(t)+E\|v(t)\|_{\rho+2}^{\rho+2}+E\|\nabla v(t)\|^{2}\right. \\
& \left.+E\|u(t)\|_{p}^{p}+E(h \circ \nabla u)(t)+E\|\nabla u(t)\|^{2}\right] .
\end{aligned}
$$

Combining (3.33) and (3.37)

$$
L^{\prime}(t) \geq K L^{\frac{1}{1-\alpha}}(t), \quad \forall t \geq 0
$$

with a positive constant $K$ depending on $C$ and $\delta \gamma$, it follows that

$$
L^{\frac{\alpha}{1-\alpha}}(t) \geq \frac{1-\alpha}{(1-\alpha) L^{-\frac{\alpha}{1-\alpha}}(0)-\alpha K t} .
$$

Let

$$
T_{0}=\frac{1-\alpha}{\alpha K L^{\frac{\alpha}{1-\alpha}}(0)} .
$$

Then $L(t) \rightarrow \infty$ as $t \rightarrow T_{0}$. This means that there exists a positive time $T^{*} \in\left(0, T_{0}\right]$ such that

$$
\lim _{t \rightarrow T^{*}} E[F(t)]=+\infty
$$

As for the case when $P\left(\tau_{\infty}=+\infty\right)<1$ (i.e., $P\left(\tau_{\infty}<+\infty\right)>0$ ), then $\|\nabla u(t)\|$ blows up in finite time $T^{*} \in\left(0, \tau_{\infty}\right)$ with positive probability. Thus, the proof of Theorem 3.1 is completed.

\section{Global existence}

In this section, we show that the solution of (1.1) is global if $q \geq p$. We use the BorelCantelli lemma to prove the existence of a global solution. For this goal, we introduce an energy function

$$
e(u(t))=\left\|u_{t}(t)\right\|_{\rho+2}^{\rho+2}+\|\nabla u(t)\|^{2}+\left\|\nabla u_{t}(t)\right\|^{2}+\|u(t)\|_{p}^{p}+(h \circ \nabla u)(t)
$$

Theorem 4.1 Assume that $\left(u_{0}, u_{1}\right) \in H_{0}^{1}(D) \times L^{2}(D), E \int_{0}^{T}\|\sigma(t)\|^{2} d t<\infty$, and condition (2.1) holds. If $q \geq p, u(t)$ is a solution of (1.1) with the initial data $\left(u_{0}, u_{1}\right) \in H_{0}^{1}(D) \times L^{2}(D)$ according to Definition 2.1 on the interval $[0, T]$, then for any $T>0$, we have

$$
E \sup _{0 \leq t \leq T} e(u(t))<\infty
$$


Proof For any $T>0$, we will show that $u_{N}(t)=u\left(t \wedge \tau_{N}\right) \rightarrow u(t)$ (a.e.) as $N \rightarrow \infty$ for any $t \leq T$, so that the local solution becomes a global solution where $\tau_{N}$ is a stopping time which is defined in Section 3. Similarly to Theorem 12 of [23], we can derive the proof of the theorem.

\section{Funding}

This work was supported by the National Research Foundation of Korea (Grant \# NRF-2016R1D1A1B03930361). This work was supported by the National Research Foundation of Korea (NRF) grant funded by the Ministry of Science, ICT and Future Planning (No. 2017R1E1A1A03070224).

\section{Abbreviations}

Stochastic quasilinear viscoelastic wave equation.

Availability of data and materials

No availability.

\section{Ethics approval and consent to participate}

All parts in this manuscript were approved by the authors.

\section{Competing interests}

The authors declare to have no competing interests.

\section{Consent for publication}

This work was consent for publication.

\section{Authors' contributions}

The article is a joint work of the three authors, who contributed equally to the final version of the paper. All authors read and approved the final manuscript.

\section{Author details}

${ }^{1}$ Hankuk University of Foreign Studies, Yongin, South Korea. ${ }^{2}$ Department of Mathematics, Pusan National University, Busan, South Korea. ${ }^{3}$ Institute of Liberal Education, Catholic University of Daegu, Gyeongsan, South Korea.

\section{Publisher's Note}

Springer Nature remains neutral with regard to jurisdictional claims in published maps and institutional affiliations.

Received: 16 August 2017 Accepted: 15 January 2018 Published online: 26 January 2018

\section{References}

1. Pazy, A: Semigroups of Linear Operators and Applications to Partial Differential Equations. Springer, New York (1983)

2. Lions, JL: Quelques Méthodes de Résolution des Problémes aux Limites Non Linéaires. Dunod, Paris (1969)

3. Liu, W: General decay and blow-up of solution for a quasilinear viscoelastic problem with nonlinear source. Nonlinear Anal. 73, 1890-1904 (2010)

4. Song, H: Global nonexistence of positive initial energy solutions for a viscoelastic wave equation. Nonlinear Anal. 125, 260-269 (2015)

5. Hao, J, Wei, H: Blow-up and global existence for solution of quasilinear viscoelastic wave equation with strong damping and source term. Bound. Value Probl. 2017, 65 (2017)

6. Chow, PL: Stochastic wave equations with polynomial nonlinearity. Ann. Appl. Probab. 12, 361-381 (2002)

7. Chow, PL: Nonlinear stochastic wave equations: blow-up of second moments in $L_{2}$-norm. Ann. Appl. Probab. 19, 2039-2046 (2009)

8. Bo, LJ, Tang, D, Wang, YG: Explosive solutions of stochastic wave equations with damping on $R^{d}$. J. Differ. Equ. 244 170-187 (2008)

9. Chow, PL: Asymptotics of solutions to semilinear stochastic wave equations. Ann. Appl. Probab. 16, 757-789 (2006)

10. Chow, PL: Asymptotic solutions of a nonlinear stochastic beam equation. Discrete Contin. Dyn. Syst., Ser. B 6, 735-749 (2006)

11. Brzeźniak, Z, Maslowski, B, Seidler, J: Stochastic nonlinear beam equations. Probab. Theory Relat. Fields 132, 119-149 (2005)

12. Carmona, R, Nualart, D: Random non-linear wave equation: smoothness of solutions. Probab. Theory Relat. Fields 95, 87-102 (1993)

13. Crauel, H, Debussche, A, Flandoli, F: Random attractors. J. Dyn. Differ. Equ. 9, 307-341 (1997)

14. Dalang, R, Frangos, N: The stochastic wave equation in two spatial dimensions. Ann. Probab. 26(I), 187-212 (1998)

15. Duncan, T, Pasik-Duncan, B: A direct approach to linear-quadratic stochastic control. Opusc. Math. 37, 821-827 (2017)

16. Just, A, Stempień, Z: Pareto optimal control problem and its Galerkin approximation for a nonlinear one-dimensional extensible beam equation. Opusc. Math. 36, 239-252 (2016)

17. Millet, A, Morien, PL: On a nonlinear stochastic wave equation in the plane: existence and uniqueness of the solution. Ann. Appl. Probab. 11, 922-951 (2001)

18. Pardoux, E: Equations aux derivées partielles stochastiques nonlinéaries monotones. Thèse, Université Paris XI (1975) 
19. Parshad, RD, Beauregard, MA, Kasimov, A, Said-Houari, B: Global existence and finite blow-up in a class of stochastic nonlinear wave equations. Commun. Stoch. Anal. 8(3), 381-411 (2014)

20. Kim, JU: On the stochastic wave equation with nonlinear damping. Appl. Math. Optim. 58, 29-67 (2008)

21. Barbu, V, Prato, GD, Tubaro, L: Stochastic wave equations with dissipative damping. Stoch. Process. Appl. 117, 1001-1013 (2007)

22. Da Prato, G, Zabczyk, J: Stochastic Equations in Infinite Dimensions. Cambridge University Press, Cambridge (1992)

23. Cheng, S, Guo, Y, Tang, Y: Stochastic viscoelastic wave equations with nonlinear damping and source terms. J. Appl. Math. 2014, Article ID 450289 (2014)

24. Gao, H, Guo, B, Liang, F: Stochastic wave equations with nonlinear damping and source terms. Infin. Dimens. Anal. Quantum Probab. Relat. Top. 16(2), Article ID 1350013 (2013)

25. Higham, DJ: An algorithmic introduction to numerical simulation of stochastic differential equations. SIAM Rev. 43(3), 525-546 (2001)

26. Wei, T, Jiang, Y: Stochastic wave equations with memory. Chin. Ann. Math., Ser. B 31(3), 329-342 (2010)

\section{Submit your manuscript to a SpringerOpen ${ }^{\circ}$} journal and benefit from:

- Convenient online submission

$\checkmark$ Rigorous peer review

- Open access: articles freely available online

- High visibility within the field

- Retaining the copyright to your article

Submit your next manuscript at $\boldsymbol{~ s p r i n g e r o p e n . c o m ~}$ 